\title{
The cochlear amplifier as a standing wave: "Squirting" waves between rows of outer hair cells?
}

\author{
Andrew Bella) \\ Research School of Biological Sciences, Australian National University, Canberra 0200, Australia \\ Neville H. Fletcher \\ Research School of Physical Sciences and Engineering, Australian National University, Canberra 0200, \\ Australia
}

(Received 15 February 2004; revised 5 May 2004; accepted 7 May 2004)

\begin{abstract}
This paper draws attention to symmetric Lloyd-Redwood (SLR) waves-known in ultrasonics as "squirting" waves-and points out that their distinctive properties make them well-suited for carrying positive feedback between rows of outer hair cells. This could result in standing-wave resonance-in essence a narrow-band cochlear amplifier. Based on known physical properties of the cochlea, such an amplifier can be readily tuned to match the full 10-octave range of human hearing. SLR waves propagate in a thin liquid layer enclosed between two thin compliant plates or a single such plate and a rigid wall, conditions found in the subtectorial space of the cochlea, and rely on the mass of the inter-plate fluid interacting with the stiffness of the plates to provide low phase velocity and high dispersion. The first property means SLR wavelengths can be as short as the distance between rows of outer hair cells, allowing standing wave formation; the second permits wide-range tuning using only an order-of-magnitude variation in cochlear physical properties, most importantly the inter-row spacing. Viscous drag at the two surfaces potentially limits SLR wave propagation at low frequencies, but this can perhaps be overcome by invoking hydrophobic effects. (C) 2004 Acoustical Society of America. [DOI: 10.1121/1.1766053]
\end{abstract}

PACS numbers: 43.64.Bt, 43.64.Kc, 43.20.Ks, 87.18.Ed [BLM] Pages: 1016-1024

\section{INTRODUCTION}

A major unresolved problem in cochlear mechanics is a basic one: how is it physically possible to finely tune the human cochlea over three decades of frequency? The conventional model involving "traveling" waves propagating lengthwise along the basilar membrane (BM) certainly gives broad tuning, with the local resonance frequency being determined largely by the plate-stiffness and width of the BM, but a local-resonance theory, in some way involving the active outer hair cells (OHCs), appears necessary to provide the observed sharp tuning. The nature of this active tuning has been a matter for speculation and debate, since the identified material properties of the cochlear structures do not vary by the large factor required in order to cover the large frequency range involved. ${ }^{1}$

Here a solution is proposed involving standing-wave resonance between the rows of OHCs. The resulting wave direction is across the partition (radially) in a direction at right angles to the standard lengthwise (longitudinal) direction of propagation of the traveling wave. If the OHCs are excited by such a traveling wave, then their mechanical responses will deflect the membrane to which they are attached and launch a secondary wave from each cell. These secondary waves will interact with the other OHCs, causing them to respond with further waves, and so on. Because the phase change of the primary exciting wave along the rows is small, many OHCs will respond in unison. Furthermore, since the OHCs are arranged in three parallel rows, positive feedback

${ }^{\text {a)} E l e c t r o n i c ~ m a i l: ~ a n d r e w . b e l l @ a n u . e d u . a u ~}$ at a resonance frequency related to the $\mathrm{OHC}$ spacing will occur and, as a result, will launch a "radial" wave in a direction normal to the rows. This mechanism would operate most efficiently if the central row, $\mathrm{OHC} 2$, responded in antiphase to the other two rows, and if response sensitivity of the individual cells were adjusted neurally to be just below the oscillation threshold.

A major difficulty confronting this radial wave hypothesis, however, is the extremely low wave velocity and high dispersion required in order to have the wavelength match the separation between $\mathrm{OHC}$ rows over the full frequency range of the human cochlea. In this paper a wave type is identified that meets these requirements: a symmetric LloydRedwood (SLR) wave, known in ultrasonics as a "squirting" wave. This mechanism appears to provide the "self-tuned critical oscillators" whose existence has been proposed on general grounds by Duke and Jülicher. ${ }^{2,3}$

\section{SLR "SQUIRTING" WAVES}

SLR waves arise when a thin fluid layer is sandwiched between two deformable plates. They were predicted by Lloyd and Redwood ${ }^{4}$ in 1965 and first experimentally verified in the ultrasonic regime by Hassan and Nagy ${ }^{5}$ in 1997. Unlike normal flexural or shear waves in a plate, ${ }^{6}$ the SLR wave relies primarily upon interaction between the inertia of the fluid and the elastic restoring force of the plates. While the original analysis of Lloyd and Redwood assumed that the plates deformed by shear, plates thinner than about one-sixth of the wavelength will deform by bending, the case considered by Coulouvrat et al. ${ }^{7}$ and by Hassan and Nagy. ${ }^{5}$ Both 


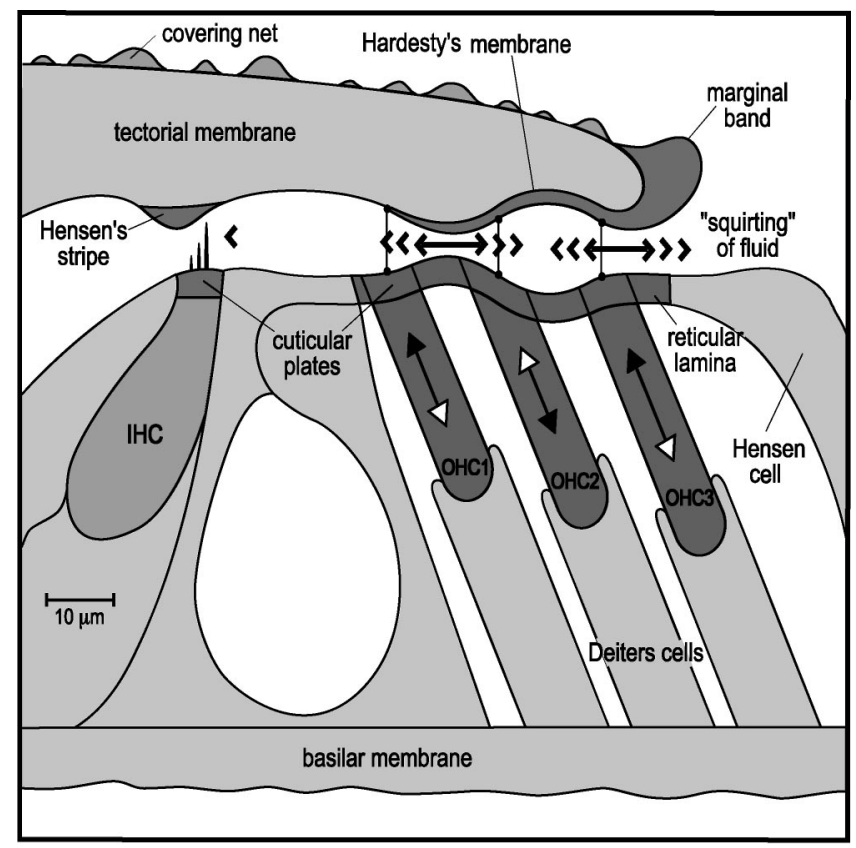

FIG. 1. Simplified diagram of the anatomy of the human cochlea in cross section. Radial SLR waves ("squirting" waves) could be generated by cyclic length changes of OHCs. Symmetric undulations induced in the facing surfaces of the TM and the RL squeeze the intervening fluid and produce a squirting action (horizontal arrows). The wave will continue to the IHCs, where squirting will tilt the free-standing IHC stereocilia. Shorter OHC stereocilia (unattached to TM and also subject to squirting effects) are not shown. White areas are occupied by fluid.

these cases are treated in Appendix A and illustrated in Fig. 3 , and certain other variations are also discussed.

To visualize liquid displacement patterns, Lloyd and Redwood solved the equations of motion numerically for two modes, one antisymmetric and the other symmetric with respect to a plane along the center of the fluid layer. In the antisymmetric mode, discussed in more detail in Appendix A, the upper and lower layers, and the fluid, move up and down together in a sinuous fashion, so that the width of the fluid layer is constant and no enhanced motion of fluid occurs. Applied to the cochlea, the lack of such fluid motion implies that the stereocilia would not be deflected. Moreover, this mode does not give appropriately low propagation speeds or such high dispersion [see (A13) in Appendix A], so we conclude it is not auditorily relevant.

The symmetric mode, however, in which the two facing solid layers vibrate in mirror symmetry to give a varicose wave, which we call the SLR mode, is of considerably greater interest. This mode involves squeezing of the intervening fluid backwards and forwards in the direction of propagation. Hassan and Nagy called it a "squirting" mode because horizontal displacements of the fluid become magnified when the gap is narrow relative to the wavelength, as is the case in the typical cochlear configuration shown in Fig. 1. Maximum horizontal velocity of fluid occurs one-quarter of a wavelength away from the place where the plates undergo maximum vertical displacement.

Hassan and Nagy studied the waves at ultrasonic frequencies $(15-150 \mathrm{kHz})$ with a liquid film approaching $1 \mathrm{~mm}$ in thickness. At audio frequencies, however, the effect of viscosity becomes increasingly pronounced (see Appendices), a factor that, acting in the subtectorial space, would tend to damp the wave and prevent its propagation unless some other mechanism intervenes. As it happens, there appears to be just such a possibility deriving from the properties of hydrophobic surfaces, as will be discussed later.

Anatomically, the cochlea has a thin layer of fluid (aqueous endolymph) enclosed between the gelatinous tectorial membrane (TM) and the thin reticular lamina (RL), as shown in Fig. 1. The two surfaces are held apart by the stereocilia of the OHCs, with the tips of the tallest stereocilia embedded in the lower surface of the TM. From the analysis of Lloyd and Redwood $^{4}$ and of Hassan and Nagy, ${ }^{5}$ the phase velocity $c$ of the symmetric Lloyd-Redwood wave for two identical plates of half-thickness $h$, Young's modulus $E$, and Poisson's ratio $\sigma$, separated by a liquid layer of thickness $d$ and density $\rho$, is given approximately by

$$
c \approx\left[\frac{E h^{3} d \omega^{4}}{3\left(1-\sigma^{2}\right) \rho}\right]^{1 / 6},
$$

provided the plates are thin compared with the wavelength so that they deform by bending. The wavelength $\lambda=2 \pi c / \omega$ is then given by

$$
\lambda \approx 2 \pi\left[\frac{E h^{3} d}{3\left(1-\sigma^{2}\right) \rho}\right]^{1 / 6} \omega^{-1 / 3},
$$

which more readily illustrates the dispersive properties of the wave. A doubling of wavelength, for example, is accompanied by an eightfold change in frequency.

Plates thicker than about one-sixth of the wavelength undergo shear instead of bending, and the approximate result for the case where the plates are still thinner than the enclosed liquid layer is

$$
c \approx\left[\frac{E h d \omega^{2}}{(1+\sigma) \rho}\right]^{1 / 4} .
$$

The corresponding expression for wavelength $\lambda$ is

$$
\lambda \approx 2 \pi\left[\frac{E h d}{(1+\sigma) \rho}\right]^{1 / 4} \omega^{-1 / 2} .
$$

As shown in Appendix A, both (1) and (3) can be simply derived by neglecting the mass of the plates and equating the kinetic energy of the "squirting" liquid to the elastic strain energy of the plates. Inclusion of the mass of the plates is simple, but complicates the resulting expressions unnecessarily.

When one of the plates is much thicker, much stiffer, or much denser than the other, then it moves very little and the motion reduces essentially to that of the original model with the immobile plate located along the center-plane of the original fluid layer. Appendix A shows that this does not change the form of the dispersion relations (1) and (3), except that $d$ is now equal to twice the thickness of the liquid layer. The wave of relevance is therefore that involving bending and with a dispersion relation of the form (1), provided at least one of the plates is sufficiently thin.

In the case of the cochlea, there is liquid on the outer side of each plate as well as between them, and the wave 
motion extends some distance into this liquid. But again, Appendix A shows that the effect of this surrounding liquid is small in the case of a structure with dimensions typical of the cochlea.

An important property of Eqs. (1) and (3) is that the SLR wave velocity increases markedly with frequency, as $\omega^{2 / 3}$ in the first case and as $\omega^{1 / 2}$ in the second. The wave is thus highly dispersive and, as given by (2) or (4), the wavelength range for a given frequency range is greatly compressed, varying as $\omega^{-1 / 3}$ and $\omega^{-1 / 2}$, respectively, for the two cases discussed, rather than as $\omega^{-1}$ for nondispersive propagation. It is this feature that potentially allows SLR waves to provide a way of tuning an active cochlear amplifier over a 3-decade (10-octave) frequency range by requiring only an order of magnitude variation in other physical parameters.

\section{SLR WAVE IN THE COCHLEA}

As Appendix A makes clear, the primary requirement for generating SLR waves in accordance with (1) is that at least one of the two enclosing plates is thin enough to deform by bending. Given the extreme thinness of the RL $(1-3 \mu \mathrm{m})$, this condition appears likely to be met in the cochlea, although no direct measurements of this structure's stiffness have been made. It is known, however, that this articulated mesh of interlocking plates appears more flexible than the basilar membrane ${ }^{8}$ and there is some indication ${ }^{9}$ that it is more compliant than the TM. In what follows, therefore, it is assumed that deformation is by bending of at least one of the plate structures involved, so that the dispersion relation is given by (1). A difficulty, however, is that no individual data set provides all required values, so it is necessary to use data compiled from measurements on several different species of mammal.

The most comprehensive data in the literature relates to the water buffalo; ${ }^{10}$ here the thicknesses of the TM (3-8 $\mu \mathrm{m})$ and RL $(1.8-2.9 \mu \mathrm{m})$ are tabulated along the cochlea. It is immediately apparent that, in this case, both of these key structures are appreciably thinner than a wavelength, suggesting that both undergo bending. Since the RL appears to have an elastic modulus comparable to that of the TM, ${ }^{9}$ it is appropriate to use RL dimensions and to combine these with a representative Young's modulus of $2 \mathrm{kPa}$, as derived from recent measurements ${ }^{11}$ on the guinea pig TM in which figures of $0.7-3.9 \mathrm{kPa}$ were reported. The gap width, $d$, reflects the height of the tallest stereocilia, and here there is no water buffalo data; instead, human data, ${ }^{12}$ showing a gradation of 3-7 $\mu \mathrm{m}$ from base to apex, are used.

For the mid-region of the cochlea where frequencies near $1000 \mathrm{~Hz}\left(\omega \approx 6000 \mathrm{rad} \mathrm{s}^{-1}\right)$ are detected, the assumed values are thus, $E \approx 2 \mathrm{kPa}, h \approx 1 \mu \mathrm{m}, d \approx 3 \mu \mathrm{m}$, and $\rho \approx 1000 \mathrm{~kg} / \mathrm{m}^{3}$. Equation (1) then gives a wave speed $c$ $\approx 40 \mathrm{~mm} / \mathrm{s}$ and a wavelength, $c / f$, of about $40 \mu \mathrm{m}$. A plot of wave speed against frequency over the length of the cochlea is shown as the full line in Fig. 2 and shows values ranging from $3 \mathrm{~mm} / \mathrm{s}$ at the apex $(20 \mathrm{~Hz})$ to $300 \mathrm{~mm} / \mathrm{s}$ at the base $(20$ $\mathrm{kHz}$ ). Extremely slow wave speeds and short wavelengths thus appear possible in the cochlear structure. According to LePage, ${ }^{13}$ the tonotopic mapping (for humans) of frequency $f$

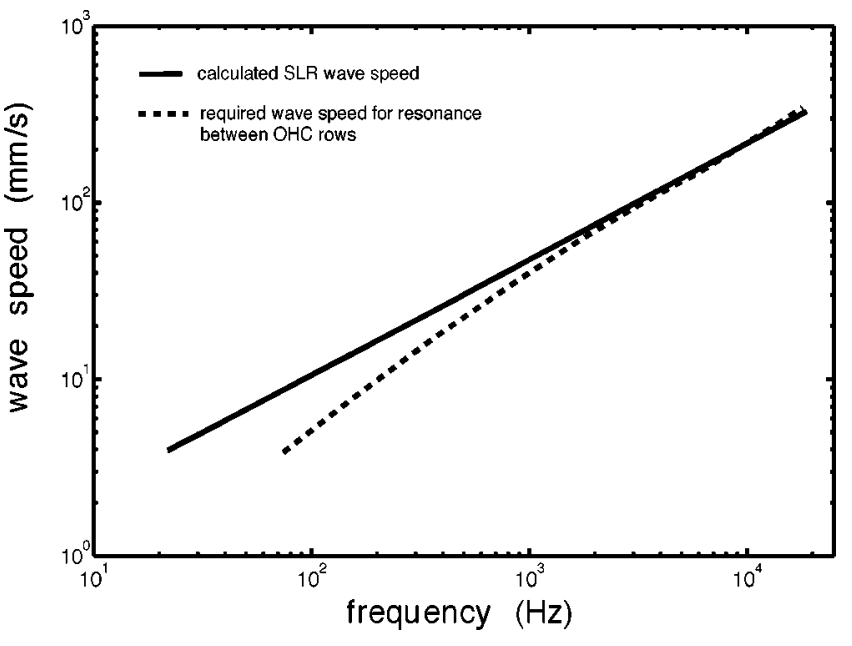

FIG. 2. Calculated speeds of SLR waves (full line) based on measured RL dimensions of the water buffalo (Ref. 10) and assuming that the RL has an elastic modulus similar to that of the TM (Ref. 11) (2 kPa). The gap width is that for human stereociliar height (Ref. 12) and a human frequency-place map (Ref. 13) is used. These speeds agree well with wave speeds inferred (dotted line) from assuming one wavelength of a standing wave to form between the experimentally determined (Ref. 17) spacing $\mathrm{OHC} 1-\mathrm{OHC} 3$ for humans.

to fractional distance $x$ from the apex is well-approximated by the function $f=165.4\left(10^{2.1 x}-0.88\right)$, and this expression is used in the following calculations.

Since Fig. 2 reflects a mixture of cochlear properties from water buffalo, guinea pig, and human, one may question the aptness of the values derived from (1) to human hearing. In general, micrographs show that the differences between these species are not major, and most cross sections appear similar. Although detailed measurements of human TM dimensions are lacking, it does seem, however, that the thickness of the human TM is, at least at the apex, appreciably greater than in the water buffalo, and its overall structure thus appears as in Fig. 1. The analysis in Appendix A then shows that SLR waves will propagate by bending of the RL with the TM remaining nearly inactive. Since the speed of an SLR wave varies only as the sixth root of the Young's modulus, errors introduced by assuming values of RL stiffness about equal to those of the TM ( $2 \mathrm{kPa})$ should not be serious.

As well as the subtectorial space in the cochlea being well-configured for propagation of SLR waves, it is important to note that $\mathrm{OHCs}$ appear strategically positioned to generate these waves, as shown in Fig. 1. A key property of OHCs is that they are electromotile, with the ability to change length, cycle by cycle, in response to variations in cell potential, ${ }^{14}$ such as might be caused by stereocilia deflection. Thus, changes in length of OHCs could excite SLR waves.

OHCs are clamped at the bottom by Deiters cells, which rest on the basilar membrane, and are firmly connected at the top to the interlocking platelike network of the RL. When $\mathrm{OHCs}$ are electrically stimulated in vivo, the RL at the top moves 5-10 times more ${ }^{8}$ than does the basilar membrane at the bottom, a key indicator that the RL is highly flexible and could readily respond to elongation and contraction of OHCs.

From this numerical analysis, supported by the theoret- 
ical results in Appendix A, it can be concluded that audiofrequency SLR waves with speeds as low as tens to hundreds of millimeters per second and wavelengths of tens to hundreds of micrometers could occur in many, if not all, mammalian cochleas. The wave speed will be governed by the bending of the thinnest membrane, usually the RL, although deformation of the TM may also contribute in some cases.

The possible existence of such radial waves prompts the question of how they may interact with a longitudinal traveling wave. Some kind of direct coupling of excitation from the longitudinal direction to the radial would presumably help in funneling energy of a particular frequency to its appropriate location on the partition, at which point the OHCs could then begin actively fine-tuning the response via SLR waves. The precise micromechanics of this process is beyond the scope of this paper. It is reasonable, however, to treat the radial wave as an independent entity because its wavelength is generally very small-tens of micrometers-compared to that of a traveling wave, which is typically in the range of millimetres. In turn this means that the input stimulus to the $\mathrm{OHCs}$ is essentially in phase over reasonably large $\mathrm{OHC}$ aggregates. In the case of spontaneous emissions, of course, where the active process dominates, the situation could be rather different.

A simple interpretation, then, broadly in keeping with existing traveling wave theory, might be that the traveling wave is the primary filter and the SLR wave the second filter. However, the SLR mechanism proposed here does underscore the importance of clarifying the nature of the primary input to the OHCs, which is not certain. In particular, the possible role of the fast pressure wave in stimulating OHCs requires careful consideration. ${ }^{15,16}$

\section{DISPERSION AND TONOTOPIC TUNING}

It was noted earlier that SLR waves are highly dispersive $\left(c \propto \omega^{2 / 3}\right)$, so that in order to vary tuning 1000-fold, dispersion will provide a factor of 100 , leaving only a factor of 10 to be contributed by other variables. This means that if inter-row spacing of OHCs were constant between base and apex, physical and geometrical characteristics of the cochlea would only be called on to alter wave speed by tenfold in order to maintain a full wavelength between $\mathrm{OHC} 1$ and $\mathrm{OHC} 3$. In reality, the spacing of $\mathrm{OHC}$ rows in humans ${ }^{17}$ widens by a factor of 2.5 from base to apex, meaning that wave speed need only vary by a factor of 4 through the other parameters in (1).

The same equation indicates that elasticity $E$ and gap thickness $d$ are of little consequence in tuning, as phase velocity only varies as their sixth root. The most likely parameter leading to tuning is the membrane half-thickness $h$, since $c \propto h^{1 / 2}$. A systematic variation in $h$ from base to apex might therefore be expected, with $h$ smaller at the apex (low frequencies). The detailed water buffalo data ${ }^{10}$ confirms this expectation. For this animal, the thickness of the TM decreases from $26 \mu \mathrm{m}$ at the base to $10 \mu \mathrm{m}$ at the apex (2.6fold); similarly, the RL thins out from $2.9 \mu \mathrm{m}$ to $1.8 \mu \mathrm{m}$ (1.6-fold).

\section{THE COCHLEAR AMPLIFIER AS A STANDING WAVE?}

Distinctive features of SLR waves are their low speeds and correspondingly short wavelengths. At the same time, a system in which motile elements (OHC cell bodies) are in close proximity to sensory elements (OHC stereocilia) immediately raises the possibility of feedback. Over the span of a single SLR wavelength the phase of a propagating wave changes by $360^{\circ}$, a situation inviting positive feedback and, given a suitable two-way interaction, standing waves. It appears significant that $\mathrm{OHCs}$ typically lie in three well-defined rows and are graded in their separation along the cochlea so as to span a distance ranging from 20 to $50 \mu \mathrm{m}$, dimensions comparable to calculated SLR wavelengths. It is also of some reassurance for our previous pooling of data that the graded spacings of $\mathrm{OHC}$ rows for both human ${ }^{17}$ and water buffalo $^{10}$ are nearly identical.

A real possibility, therefore, is that positive feedback may occur between $\mathrm{OHC}$ rows. In response to a sound stimulus, the OHCs will undergo movement, launching an SLR wave, and the distinctive squirting motion of the wave will then initiate positive feedback through bending of neighboring OHC stereocilia, creating a standing wave. Here we consider that it is the shorter $\mathrm{OHC}$ stereocilia, which are freestanding, that are bent and contribute most to feedback. At the same time, the tallest stereocilia, which are firmly attached to the TM may still contribute feedback as they must tilt with respect to their bases when the reticular lamina, on which they rest, undulates underneath. The important result is that in the end some of the oscillating fluid flow associated with the standing wave will escape the $\mathrm{OHC}$ region and propagate towards the IHCs, where the jetting fluid will bend stereocilia (which here are all free-standing) and greatly enhance the responses of the cells at the standing-wave resonance frequency.

A mention of nonradial propagation of SLR waves is also called for. Because OHCs are regularly arranged longitudinally as well as radially, cell interaction may launch lengthwise SLR waves, too. We note, however, that the longitudinal cell spacing is smaller than the radial spacing, so the corresponding resonance frequency would be much higher, perhaps making the initial tuned stimulus from a traveling wave ineffective. Moreover, these waves would travel in directions that would not strongly affect the IHCs. While subtle effects due to nonradial waves cannot therefore be immediately ruled out, they do not constitute the major mechanism investigated here.

The location of the maxima and minima of the standing wave relative to the $\mathrm{OHC}$ rows will depend upon the mechanical impedance of the OHCs relative to the wave impedance of the surrounding plate. Since the cells are large in diameter relative to the thickness of the plate, it is likely that their mechanical impedance (force divided by displacement velocity) is also relatively large, which means that the standing wave will be excited in such a way that the OHCs lie close to, but not coincident with, the displacement nodes of the plate. Furthermore, because these plate displacement nodes are also the regions of maximum squirting wave fluid velocity (and maximum tilt of stereocilia with respect to their 
bases), this location also provides optimal feedback to the OHCs through displacement of their stereocilia. Although each $\mathrm{OHC}$ acts as a circular wave source, their linear arrangement effectively produces a nearly linear wavefront parallel to the $\mathrm{OHC}$ rows, and in this way an escaping wave propagates at right angles to the rows and towards the IHCs. Some experiments ${ }^{18,19}$ have seen large phase variations across the partition (up to $180^{\circ}$ between points $10 \mu \mathrm{m}$ apart ${ }^{18}$ ), which can be interpreted as good evidence for short wavelength radial wave motion; however others ${ }^{20}$ have seen no radial phase variability, so that more work is needed to clarify this behavior.

The dotted line in Fig. 2 shows the phase velocity required to create feedback resonance between rows of OHCs in the human cochlea, placed next to a line showing the wave velocities expected from an SLR wave based on composite cochlear data. To calculate the dotted line, the speed needed to make the $\mathrm{OHC} 1-\mathrm{OHC} 3$ distance a full-wavelength standing wave cavity was used; this distance is continuously graded ${ }^{17}$ from base $(20 \mu \mathrm{m})$ to apex $(50 \mu \mathrm{m})$ in humans, and the same frequency-place map ${ }^{13}$ was again used to convert location to frequency. The general trend and proximity of the lines support the possibility that resonance between $\mathrm{OHC}$ rows may occur via SLR waves. An SLR wave thus makes an ideal candidate for tuning standing waves between $\mathrm{OHC}$ rows. Modeling of this process is incomplete, and so further details are not given here. However, since OHC stereocilia are particularly sensitive to lateral jets of fluid, ${ }^{21}$ the postulated reverberating activity between rows of $\mathrm{OHCs}$ could provide a physical realization of the cochlear amplifier, the device proposed by Davis ${ }^{22}$ to explain the active nature of the cochlea at low sound pressures. It also has strong parallels with the "regenerative receiver" described by Gold ${ }^{23}$ and with surface acoustic wave (SAW) resonator devices. ${ }^{15,16}$ If SLR waves do operate in the cochlea as supposed here, it would confirm some long-standing conjectures that fluid flow in the subtectorial space was crucial for IHC stimulation $^{24,25}$ and would relate to a recent speculation ${ }^{26}$ that the cochlear amplifier was a fluid pump.

There is, however, a major problem with the SLR wave hypothesis: the analysis in Appendix A indicates that propagation of SLR waves in the narrow subtectorial space might be expected to be strongly damped by viscous forces, particularly at low frequencies as indicated in Eq. (A15). But it is now known that the effects of viscosity in narrow channels can be greatly diminished when hydrophobic surfaces are involved, and it may well be that the cochlea makes use of this phenomenon. As described in more detail in Appendix $\mathrm{B}$, slippage between a polar liquid and its bounding surfaces can be considerably enhanced if the surfaces are made hydrophobic by coating them with a thin layer of oil. The relevance here is that lipid droplets are secreted by Hensen cells, immediately next to the subtectorial space (see Fig. 1), and a natural supposition is that the function of these droplets is to coat both TM and RL surfaces (but presumably not the stereocilia) and so reduce their viscous drag upon the squirting fluid in the subtectorial space.

\section{CONCLUSIONS}

This paper has constructed an attractively simple model for sharp tuning in the cochlea by assuming that SLR waves are generated by interaction between rows of motile outer hair cells, the reticular lamina, and the fluid lying between it and the tectorial membrane. In turn, these squirting waves create, through stereocilia-mediated positive feedback, a standing wave between the rows. The gain of the reverberating system-operating broadly like a solid-state surface acoustic wave device-is presumably neurally adjusted so as to be close to the oscillation threshold in order to provide high gain and narrow frequency response. Squirting waves generated in the $\mathrm{OHC}$ region could propagate radially across the space to the inner hair cells and there initiate a strong and sharply tuned neural response.

This model also displays other interesting features. For example, it assigns a role to Hensen cell lipids in overcoming limitations imposed by viscosity. It also points to a highly localized basis for the cochlear amplifier, suggesting for example that spontaneous otoacoustic emissions could arise from a small group of $\mathrm{OHCs}$ with positive feedback gain exceeding the oscillation threshold for SLR waves.

\section{ACKNOWLEDGMENTS}

The authors thank M. V. Srinivasan, A. W. Gummer, and T. Maddess for helpful comments. A.B. is supported by a Ph.D. scholarship from the Australian National University and received seed funding from the University of Tübingen. This research complies with the Declaration of Helsinki; a full statement of animal research ethics for A.B. is set out elsewhere (Ref. 27). Catherine Eadie helped in drawing the figures.

Note added in proof. Since acceptance of this article we have come across the papers "Active control of waves in a cochlear model with subpartitions," by R. S. Chadwick, E. K. Dimitriadis, and K. H. Iwasa, Proc. Natl. Acad. Sci. U.S.A., 93, 2564-2569 (1996) and "Evidence of tectorial membrane radial mositon in a propagating mode of a comples cochlear model," by H. Cai, B. Shoelson, and R. S. Chadwick, Proc. Natl. Acad. Sci. U.S.A., 101, 6243-6248 (2004). These papers considered radial fluid motion in the RL-TM gap, but rejected it because of viscosity considerations.

\section{APPENDIX A: DERIVATION OF EQUATIONS GOVERNING SQUIRTING WAVES}

Suppose that, to conform to the notation of previous investigators, the system consists of two identical parallel plates, each of thickness $2 h$, density $\rho_{1}$, Young's modulus $E$, and Poisson's ratio $\sigma$, separated by a layer of liquid of thickness $d$, and density $\rho$, as shown in Fig. 3. The simplest way to determine the phase velocity $c$ of a wave of angular frequency $\omega$ that is symmetric about the center plane $\mathrm{AB}$, which we have called an SLR wave, is to equate the maximum values of the potential and kinetic energies of the wave. This procedure is clearly appropriate in the case of standing waves, where displacement and velocity are $90^{\circ}$ out of phase with each other, but can also be shown to be correct for propagating waves. In the derivations below, some factors of 

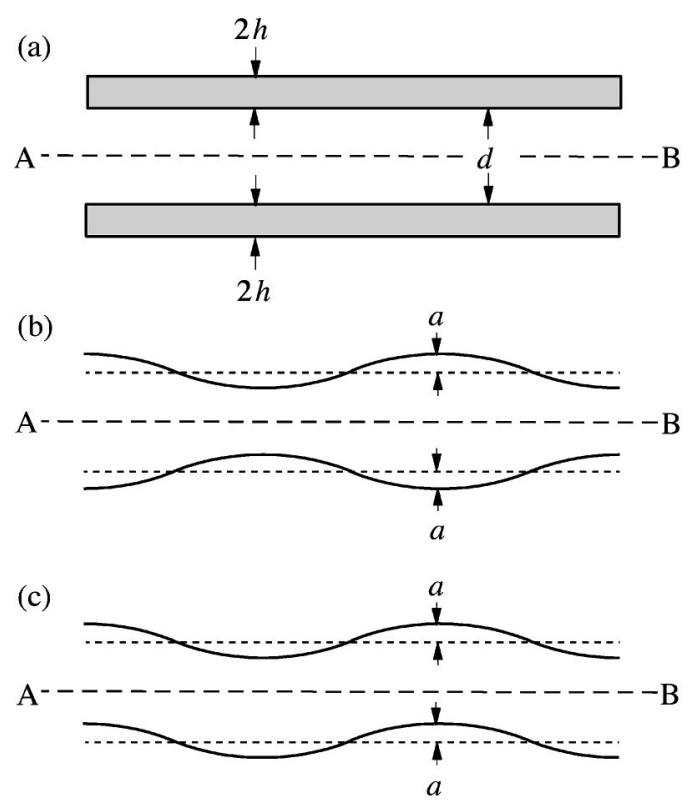

FIG. 3. (a) Section of two parallel plates surrounded by liquid. (b) Geometry of the symmetric Lloyd-Redwood (SLR) wave, the motion of which gives rise to the "squirting" of liquid between the plates. (c) The antisymmetric wave, displayed here for completeness. It is considered to play no functional role in the cochlea.

order unity are neglected in the interests of simplicity of presentation. The final results are therefore only approximate but, since fourth or sixth roots are involved, this is of little practical consequence.

In what follows, we consider the behavior on only one side of the symmetry plane $\mathrm{AB}$, and assume a standing wave of the form

$$
y(x, t)=a \cos k x \sin \omega t,
$$

where $k=\omega / c$. If $\lambda$ is the wavelength at angular frequency $\omega$, and the plates are sufficiently thin that $h \ll \lambda$, then their elastic distortion occurs through bending, and the peak elastic potential energy $P_{\text {bend }}$ per unit area is

$$
P_{\text {bend }}=\frac{E h^{3} k^{4} a^{2}}{3\left(1-\sigma^{2}\right)}=\frac{E h^{3} \omega^{4} a^{2}}{3\left(1-\sigma^{2}\right) c^{4}} .
$$

If, however, the plates are thicker so that $h$ is greater than about $\lambda / \pi$, then the plates distort predominantly by shear rather than bending, and the corresponding result is

$$
P_{\text {shear }}=\frac{E h k^{2} a^{2}}{2(1+\sigma)}=\frac{E h \omega^{2} a^{2}}{2(1+\sigma) c^{2}} .
$$

The difference in structure between (A2) and (A3) is accounted for partly by the fact that the bending modulus is involved in (A2) while the shear modulus is involved in (A3), and partly by the fact that the wave equation for a bending wave involves the operator $\partial^{4} z / \partial x^{4}$ while that for a shear wave involves only $\partial^{2} z / \partial x^{2}$.

The kinetic energy in the simple system considered involves two contributions, one from the moving mass of the plates, and one from that of the liquid between them. The kinetic energy $K_{\text {plate }}$ per unit area of the single plate has the simple form

$$
K_{\text {plate }}=\rho_{1} h \omega^{2} a^{2},
$$

but the liquid motion requires more analysis.

From (A1), if it is assumed that the plates are close enough together that $d \ll 1$, the fluid flow velocity in the space between the plates is essentially parallel to $\mathrm{AB}$ and has the form

$$
v(x, z, t)=\frac{2 f(z)}{d} \int_{0}^{x} \frac{\partial y}{\partial x} d x=\frac{2 a \omega}{k d} f(z) \sin k x \cos \omega t,
$$

where $z$ is the coordinate normal to the plates and the function $f(z)$ is approximately parabolic and becomes zero at the plane of contact with the plates, so that $\int_{0}^{d} f(z) d z=1$. Since $f(z)$ contributes a factor of order unity, it will be neglected in the following analysis. The mean square velocity amplitude averaged over the $x$-direction is

$$
\left\langle v^{2}\right\rangle \approx \frac{2 a^{2} \omega^{2}}{k^{2} d^{2}}=\frac{2 a^{2} c^{2}}{d^{2}},
$$

and the peak kinetic energy of the flow is

$$
K_{\text {liq }} \approx \frac{\rho a^{2} c^{2}}{d} .
$$

Finally, because in the case of the cochlea the plates are immersed in a surrounding liquid, account must be taken of the kinetic energy associated with flow in this liquid. Consideration of the wave equation for a liquid with a standing or propagating wave disturbance imposed upon its surface shows that this wave is exponentially attenuated with distance $y$ from the surface by a factor $\exp (-k y)$. To evaluate, to an adequate approximation, the kinetic energy associated with this motion, the quantity $d$ in (A7) can simply be replaced by $k^{-1}=c / \omega$, giving a kinetic energy contribution

$$
K_{\text {outer }}=\frac{1}{2} \rho a^{2} c \omega,
$$

and the total kinetic energy is

$$
K_{\text {total }}=K_{\text {plate }}+K_{\text {liq }}+K_{\text {outer }} \text {. }
$$

The total symmetric propagation problem can now be solved by choosing either $P_{\text {bend }}$ or $P_{\text {shear }}$, depending upon the thickness of the plates, and setting this equal to $K_{\text {total }}$. For the standard SLR-wave situation, the plates are taken to be thin enough that $h \ll \lambda / 2 \pi$ so that $P_{\text {bend }}$ is the appropriate choice, and they are close enough together that $d \ll \lambda / 2 \pi$, so that $K_{\text {plate }}$ and $K_{\text {outer }}$ can be neglected relative to $K_{\text {liq }}$. Setting $P_{\text {bend }}=K_{\text {liq }}$ then leads to the Hassan-Nagy result

$$
c \approx\left[\frac{E h^{3} d \omega^{4}}{3\left(1-\sigma^{2}\right) \rho}\right]^{1 / 6} \propto \omega^{2 / 3} .
$$

If the thickness of the plates is comparable to or greater than the wavelength, however, then distortion is by shear and, provided the plates are close enough together that $K_{\text {liq }}$ is still greater than $K_{\text {plate }}$ and $K_{\text {outer }}$, the equation $P_{\text {shear }}=K_{\text {liq }}$ leads to the result

$$
c \approx\left[\frac{E h d \omega^{2}}{(1+\sigma) \rho}\right]^{1 / 4} \propto \omega^{1 / 2} .
$$


For intermediate cases, an appropriate interpolation between (A2) and (A3) for the potential energy must be used, and the full expression (A9) may be required for the kinetic energy.

One further important implication of the model is also worth noting. The squirting-wave motions considered above are mirror-symmetric about the central plane AB of Fig. 3. This means that exactly the same results will be obtained if a rigid plate is set along this plane, so that there is only a single thin plate separated from it by a liquid-filled space of width $d / 2$. Indeed, the elastic stiffness of a thin plate increases so rapidly with its thickness, as indicated by (A2), that in most asymmetric situations an assumption that the thicker plate is essentially rigid will provide a good approximation, provided the thinner plate can distort by bending rather than shear. Of course, the relative elastic moduli of the two plates must also be taken into account.

A similar approach to that above can be applied to the antisymmetric case. Since there is no squirting motion, the enclosed fluid simply moves up and down with the enclosing plates, and its mass is added to the combined plate mass. For plates thinner than about $\lambda / 6$, so that they deform by bending, the result is

$$
c \approx\left[\frac{2 E h^{3}}{3\left(\rho d+4 \rho_{1} h\right)\left(1-\sigma^{2}\right)}\right]^{1 / 4} \omega^{1 / 2},
$$

where $2 h$ is the thickness and $\rho_{1}$ the density of each of the plates. If the plate sandwich is taken to be much thinner than $\lambda / 6$ and immersed in surrounding liquid, as discussed above for the symmetric case, then the loading effect of the surrounding evanescent waves must be taken into account. The result is a propagation law of the form

$$
c \approx\left[\frac{E h^{3}}{3\left(1-\sigma^{2}\right) \rho}\right]^{1 / 5} \omega^{3 / 5} .
$$

These equations imply much faster speed and rather less dispersion than in the symmetric case. Another point of interest is that, in the case discussed above in which one of the plates is essentially rigid and the other flexible, antisymmetric waves do not exist, as can be seen from simple symmetry considerations.

There is, however, an apparent major obstacle to this cochlear model, namely the viscosity of the liquid in the narrow region between the two plates. These viscous losses will generally exceed all other losses in the system and thus provide the primary wave damping. The viscosity $\eta$ of water at body temperature is about $7 \times 10^{-4} \mathrm{~Pa}$ s, so that the diffusion length $L \approx(\eta / \rho \omega)^{1 / 2}$ at a frequency of $1 \mathrm{kHz}$ is about $10 \mu \mathrm{m}$ and essentially all of the inter-plate liquid will be within the boundary layer. Viscosity will therefore provide a nearly frequency-independent damping force $\kappa v \approx(\eta / d) v$ per unit area, where $v$ is the flow velocity. Inserting this viscous damping term, an equation describing the behavior of an SLR standing wave has the form

$$
\rho d \frac{\partial^{2} y}{\partial t^{2}}+\frac{\eta}{d} \frac{\partial y}{\partial t}+K y=0
$$

where $y$ measures the longitudinal displacement of the fluid between the plates and $K$ is the elastic stiffness of these plates, expressed in terms of $y$. If a standing-wave resonance for this oscillation is considered, then the quality factor $Q$ is given by

$$
Q=\frac{\rho d^{2} \omega}{\eta}
$$

where $\omega$ is the frequency of the standing-wave resonance. Inserting numerical values for the human cochlea into (A14) gives $Q \approx 10^{-5} \omega$, so that at $1 \mathrm{kHz} Q$ is only about 0.1 and about 1 at $10 \mathrm{kHz}$. Any such standing-wave resonance is therefore virtually nonexistent under these simple assumptions. While active resonant feedback between cells would contribute negative resistance that could help reduce the effect of this damping, this would not overcome the damping between $\mathrm{OHCl}$ and the IHCs, so the waves could not then propagate effectively.

Propagating SLR waves of frequency $\omega$ in the system are attenuated in amplitude as $\exp (-\omega x / 2 c Q)$, which amounts to $\exp (-\pi / Q)$ per wavelength. Clearly we require that $Q>1$ for propagating waves to have any significance. Since $Q$ increases nearly linearly with frequency while the viscous barrier-layer thickness is greater than the liquid film thickness, as assumed above (and actually as the square root of frequency above this limit), this explains why SLR waves have been studied mainly at megahertz frequencies and for much thicker liquid layers than found in the cochlea.

As suggested in the main text, however, the existence of a hydrophobic film on each of the two surfaces involved could induce slip between the endolymph and its bounding surfaces in the subtectorial space, thereby overcoming this limitation. The basis of viscosity calculations is the classical "no slip" assumption, and for narrow channels and hydrophobic surfaces this is not always correct. Instead, the interface may give rise to relative slip, ${ }^{28}$ and this will make the liquid more slippery than its bulk viscosity would predict. In laboratory experiments ${ }^{29}$ the effective viscous drag was reduced by a measured factor of about 5 for films of the thickness found in the cochlea and a single treated surface, using simple laboratory chemicals to produce the film. Such a film applied to both surfaces might be expected to increase the resonant $Q$ value by a factor of about 10 , and thus to about 1 at $1 \mathrm{kHz}$ and 10 at $10 \mathrm{kHz}$, which begins to allow significant propagation of SLR waves. Indeed, when more is known about the molecular and hydrodynamic mechanisms involved, the increase might prove to be larger than this.

\section{APPENDIX B: VISCOSITY AND THE EFFECTS OF HYDROPHOBICITY}

As outlined in the main text and calculated in Appendix A, the viscosity of the waterlike endolymph between the reticular lamina and the tectorial membrane appears at first to offer an insurmountable barrier to the propagation of SLR waves below about $10 \mathrm{kHz}$.

However, the classical "no slip" assumption underlying high viscous forces in narrow channels may be unwarranted. Helmholtz ${ }^{30}$ in 1860 published an analysis of experiments 
using a water-gold interface and concluded there was appreciable slip. More recent experiments using atomic force microscopy ${ }^{31}$ have confirmed these long-held suspicions and shown that in some situations the solid surface and liquid may slip relative to each other, a phenomenon describable in terms of a "slip length." 28 The present focus of much surface physics is on understanding the unique properties of water, and it is now known that water near boundaries is more "slippery" than its bulk viscosity value would predict.

The physics underlying slippage is still uncertain, but it is clear that the effect is one involving surface tension and is greatest for hydrophobic surfaces. ${ }^{32}$ It is therefore significant for the configuration of the cochlea that Hensen cells are located immediately adjacent to the subtectorial space and that these cells are characterized by abundant production of lipid droplets ${ }^{33}$ which, at least in the case of guinea pigs, accumulate on their surface. The function of the droplets is puzzling, but one could suppose that this substance might be readily conveyed by capillary effects to the nearby reticular lamina and, via the marginal net, to the underside of the tectorial membrane. By coating the subtectorial space with an oily, hydrophobic film, the cochlea could overcome the limitations imposed by the bulk viscosity of water and be able to support a full range of audiofrequency SLR waves. Significantly, Hensen cells are larger and the lipid droplets more abundant at the low-frequency apex where, as (A15) indicates, viscosity reduction is most needed. At the same time, the height of the tallest $\mathrm{OHC}$ stereocilia (and hence the width of the subtectorial gap) increases from about $3 \mu \mathrm{m}$ at the base to $7 \mu \mathrm{m}$ at the apex, again helping to reduce viscous drag.

The evidence for hydrophobic surfaces reducing viscous forces is widespread, but it has been collected using varying apparatus under disparate conditions. Perhaps most relevant to the subtectorial space is the finding ${ }^{29}$ that the force between a spherical surface vibrating underwater within several micrometers of a plane surface was reduced by a factor of about 5 when one of the surfaces was made hydrophobic (and suggesting a factor of 10 if both surfaces were treated). Another experiment ${ }^{34}$ involving a sphere and a plane vibrating relative to each other underwater found a slip length of up to $2 \mu \mathrm{m}$ under some conditions; in this case hydrodynamic forces were 2-4 orders of magnitude less than those expected from the no-slip condition. More indirectly, observations $^{35}$ of water droplets sandwiched between hydrophobically treated glass plates have measured flow resistances $95 \%$ less than when the plates were untreated.

Much current work in surface physics is aimed at enhancing slippage between water and adjacent surfaces, either to increase water repellency or reduce hydrodynamic drag. Of particular interest, one way of increasing a surface's hydrophobicity is to increase the surface roughness, ${ }^{36}$ leading to "superhydrophobic states" with contact angles approaching $180^{\circ}$. Thus, counterintuitively, a rough surface with high surface area can exhibit appreciably less hydrodynamic drag. A standard method of increasing surface roughness is to create tiny fingerlike protrusions from a surface, ${ }^{37}$ in this way making the surface resemble that of a lotus leaf, off which water droplets effortlessly roll. In the cochlea we note that the reticular lamina ${ }^{38}$ (as well as Hensen cells ${ }^{33}$ ) is decorated with similarly shaped microvilli whose large surface area would act to increase the hydophobicity of the surfaces from which they protrude.

These considerations suggest that the ear may use hydrophobic properties to increase slippage and escape the standard limitations imposed by viscosity. Measurements of the contact angle of endolymph on the reticular lamina would be of great interest, as would modern assessments of the chemical make up and physical properties of the lipids secreted by Hensen cells, which, as far as now known, are made up of cholesterol esters, triglycerides, and phospholipids. ${ }^{39}$ These substances may provide a more effective slip than the materials used so far in surface film experiments.

${ }^{1}$ R. C. Naidu and D. C. Mountain, "Measurements of the stiffness map challenge a basic tenet of cochlear theories," Hear. Res. 124, 124-131 (1998).

${ }^{2}$ T. Duke and F. Jülicher, "Active traveling wave in the cochlea," Phys. Rev. Lett. 90, 158101 (2003).

${ }^{3}$ S. Camalet, T. Duke, F. Jülicher, and J. Prost, "Auditory sensitivity provided by self-tuned critical oscillations of hair cells," Proc. Natl. Acad. Sci. U.S.A. 97, 3183-3188 (2000).

${ }^{4}$ P. Lloyd and M. Redwood, "Wave propagation in a layered plate composed of two solids with perfect contact, slip, or a fluid layer at their interface," Acustica 16, 224-232 (1965).

${ }^{5}$ W. Hassan and P. B. Nagy, "On the low-frequency oscillation of a fluid layer between two elastic plates," J. Acoust. Soc. Am. 102, 3343-3348 (1997).

${ }^{6}$ M. C. Junger and D. Feit, Sound, Structures, and Their Interaction (Acoustical Society of America, New York, 1993), pp. 236-239.

${ }^{7}$ F. Coulouvrat, M. Rousseau, O. Lenoir, and J.-L. Izbiki, "Lamb-type waves in a symmetric solid-fluid-solid trilayer," Acust. Acta Acust. 84, 12-20 (1998).

${ }^{8}$ F. Mammano and J. F. Ashmore, "Reverse transduction measured in the isolated cochlea by laser Michelson interferometry," Nature (London) 365, 838-841 (1993).

${ }^{9}$ R. C. Naidu, "Mechanical properties of the organ of Corti and their significance in cochlear mechanics," Ph.D. thesis, Boston University, 2001, pp. 117-134.

${ }^{10} \mathrm{H}$. Tiedemann, "A new approach to theory of hearing," Acta Otolaryngol. Suppl. (Stockh) 277, 1-50 (1970).

${ }^{11}$ B. Shoelson, E. K. Dimitriadis, H. Cai, and R. S. Chadwick, "Theoretical and experimental considerations for the study of anisotropic elastic moduli of the mammalian tectorial membrane," Midwinter Meeting, Association for Research in Otolaryngology, Florida, 2003, http://www.aro.org/ archives/2003/2003_732.html

${ }^{12}$ A. Wright, "Dimensions of the cochlear stereocilia in man and the guinea pig," Hear. Res. 13, 89-98 (1984).

${ }^{13}$ E. L. LePage, "The mammalian cochlear map is optimally warped," J. Acoust. Soc. Am. 114, 896-906 (2003).

${ }^{14}$ B. N. Evans and P. Dallos, "Stereocilia displacement induced somatic motility of cochlear outer hair cells," Proc. Natl. Acad. Sci. U.S.A. 90, 8347-8351 (1993).

${ }^{15}$ A. Bell, "Are outer hair cells pressure sensors? Basis of a SAW model of the cochlear amplifier," in Biophysics of the Cochlea, edited by A. W. Gummer (World Scientific, Singapore, 2003), pp. 429-431.

${ }^{16}$ A. Bell, "Cochlear mechanics without the traveling wave: The cochlea as a surface acoustic wave resonator," preprint at http://eprints.anu.edu.au/ archive/00001258/

${ }^{17} \mathrm{G}$. Bredberg, "Cellular pattern and nerve supply of the human organ of Corti," Acta Oto-Laryngol., Suppl. 236, 1-135 (1968), Fig. 92.

${ }^{18}$ K. E. Nilsen and I. J. Russell, "Timing of cochlear feedback: Spatial and temporal representation of a tone across the basilar membrane," Nature Neurosci. 2, 642-648 (1999).

${ }^{19}$ M. P. Scherer, M. Nowotny, E. Dalhoff, H.-P. Zenner, and A. W. Gummer, "High-frequency vibration of the organ of Corti in vitro," in Biophysics of the Cochlea, edited by A. W. Gummer (World Scientific, Singapore, 2003), pp. 271-277. 
${ }^{20}$ T. Y. Ren, Y. Zou, J. F. Zheng, A. L. Nutall, E. Porsov, and S. Matthews, "Measurements of basilar membrane vibration using a scanning laser interferometer," in Biophysics of the Cochlea, edited by A. W. Gummer (World Scientific, Singapore, 2003), pp. 211-219.

${ }^{21}$ B. Canlon and L. Brundin, "Mechanically induced length changes of isolated outer hair cells are metabolically dependent," Hear. Res. 53, 7-16 (1991).

${ }^{22}$ H. Davis, “An active process in cochlear mechanics," Hear. Res. 9, 79-90 (1983).

${ }^{23}$ T. Gold, "Hearing II. The physical basis of the cochlea," Proc. R. Soc. London, Ser. B 135, 492-498 (1948).

${ }^{24}$ C. R. Steele, "A possibility for subtectorial membrane fluid motion," in Basic Mechanisms in Hearing, edited by A. R. Møller (Academic, New York, 1973), pp. 69-93.

${ }^{25}$ G. H. Frommer and C. R. Steele, "Permeability of fluid flow through hair cell cilia," J. Acoust. Soc. Am. 65, 759-764 (1979).

${ }^{26}$ K. D. Karavitaki and D. C. Mountain, "Is the cochlear amplifier a fluid pump?" in Biophysics of the Cochlea, edited by A. W. Gummer (World Scientific, Singapore, 2003), pp. 310-311.

${ }^{27}$ http://eprints.anu.edu.au/archive/00001532/

${ }^{28}$ S. Granick, Y. Zhu, and H. Lee, "Slippery questions about complex fluids flowing past solids," Nature Mater. 2, 221-227 (2003).

${ }^{29}$ W. Hild, A. Opitz, J. A. Schaefer, and M. Scherge, "The effect of wetting on the microhydrodynamics of surfaces lubricated with water and oil," Wear 254, 871-875 (2003).
${ }^{30} \mathrm{~S}$. Goldstein, "Note on the conditions at the surface of contact of a fluid with a solid body," in Modern Developments in Fluid Dynamics, edited by S. Goldstein (Dover, New York, 1965), pp. 676-680.

${ }^{31}$ V. S. J. Craig, C. Neto, and D. R. M. Williams, "Shear-dependent boundary slip in an aqueous Newtonian liquid," Phys. Rev. Lett. 87, 054504 (2001).

${ }^{32}$ C. Cotton-Bizonne, J.-L. Barrat, L. Bocquet, and E. Charlaix, "Lowfriction flows of liquid at nanopatterned interfaces," Nature Mater. 2, 237-240 (2003).

${ }^{33}$ M. A. Merchan, J. A. Merchan, and M. D. Ludena, "Morphology of Hensen's cells," J. Anat. 131, 519-523 (1980).

${ }^{34}$ Y. Zhu and S. Granick, "Rate-dependent slip of Newtonian liquid at smooth surfaces," Phys. Rev. Lett. 87, 096105 (2001).

${ }^{35}$ J. Kim and C.-J. Kim, "Nanostructured surfaces for dramatic reduction of flow resistance in droplet-based microfluidics," Proc. 2002 IEEE Conference MEMS, Las Vegas, NV, pp. 479-482 (2002).

${ }^{36}$ A. Lafuma and D. Quéré, "Superhydrophobic states," Nature Mater. 2, 457-460 (2003).

${ }^{37}$ L. Feng, S. Li, Y. Li, H. Li, L. Zhang, J. Zhai, Y. Song, B. Liu, L. Jiang, and D. Zhu, "Super-hydrophobic surfaces: from natural to artificial," Adv. Mater. (Weinheim, Ger.) 14, 1857-1860 (2002).

${ }^{38}$ R. V. Krstic, Ultrastructure of the Mammalian Cell: An Atlas (SpringerVerlag, Berlin, 1979), pp. 220-221.

${ }^{39}$ M. Schiff and H. Christiansen-Lou, "The nature of lipid globules in Hensen's cells," Ann. Otol. Rhinol. Laryngol. 76, 624-637 (1967). 